\title{
Nasal Carriage of Community Acquired and Inducible Dormant Methicillin Resistant Staphylococcus aureus among Healthcare Workers of Mansoura University Children's Hospital
}

\author{
${ }^{1}$ Gehad Mostafa, ${ }^{1}$ Mohammed Fath-Allah Badr, ${ }^{2}$ Mayada S. Zeid, ${ }^{1}$ Heba Eldegla* \\ ${ }^{1}$ Department of Medical Microbiology and Immunology, Faculty of Medicine, Mansoura University, Mansoura, Egypt \\ ${ }^{2}$ Mansoura University Children Hospital, Faculty of medicine, Mansoura University, Mansoura, Egypt
}

Key words:

MRSA; nasal colonization;

CA-MRSA; HCWs

*Corresponding Author:

Heba Elsayed Abd Elmonem

Eldegla,

Department of Medical

Microbiology and

Immunology, Faculty of

Medicine, Mansoura

University, Egypt.

Tel.: 020-1006454655

heba_degla@yahoo.com

\begin{abstract}
Background: Healthcare workers (HCWs) are at risk for Methicillin Resistant Staphylococcus aureus (MRSA) nasal carriage, subsequent infection and potential transmission of nosocomial infection. Characterization of MRSA detected in HCWs would give data that can be used for prevention and control measures. Objectives: To detect the prevalence and risk factors for MRSA nasal colonization of HCWs in Mansoura University Children's Hospital (MUCH), then determine if the sources of MRSA isolates are community or hospital acquired by detection of SCCmec IV and Panton-Valentine Leukocidin (PVL) genes. Methodology: Nasal swabs were collected from $100 \mathrm{HCWs}$ and processed to detect Staphylococcus aureus (S. aureus) strains, cefoxitin disc diffusion test of S. aureus isolates was done to select MRSA and Methicillin Sensitive Staphylococcus aureus (MSSA) strains, then all the MRSA isolates were further molecularly characterized. MSSA strains were screened for the presence of mecA gene. A second follow up swab was obtained from positive MRSA carriers in the first swab after three months. Results: The Prevalence of nasal carriage of MRSA among HCWs in MUCH was 25\% (twenty-five MRSA) isolates, four of them SCCmec IV and PVL positive CA-MRSA strains (16\%) and four MSSA (4\%) isolates were all mecA negative, none of them were inducible dormant Methicillin Resistant Staphylococcus aureus (ID-MRSA). Persistent MRSA carriers accounted for $52 \%$ of previously colonized HCWs. Conclusion: a considerable proportion of HCWs harbored CA-MRSA in their nares. Abbreviations: MRSA: Methicillin Resistant Staphylococcus aureus; HCWs: Healthcare workers; S. aureus, Staphylococcus aureus.
\end{abstract}

\section{INTRODUCTION}

Skin and soft tissue infections, food poisoning, endocarditis, osteomyelitis, and life-threatening postsurgical infections are all caused by Staphylococcus aureus. Methicillin Resistant S. aureus (MRSA), an antibiotic-resistant strain of $S$. aureus, has emerged as a major pathogen in hospitals across the world, causing treatment failure and hospital-acquired illnesses ${ }^{\mathbf{1}}$.

Healthcare workers (HCWs) play an important role in MRSA infection epidemiology ${ }^{2}$. HCWs act as vectors for transmission of MRSA as they work at the interface between hospital and the community. MRSA is most transmitted in the hospital setting by contact with HCWs' hands, clothing, or equipment. ${ }^{3}$.

Several variables influence the prevalence of MRSA colonization, including the kind of hospital department, MRSA prevalence among patients, and insufficient adherence to infection control measures; they vary based on geographical location ${ }^{4}$.
The anterior nares are the main reservoir of MRSA, although other body sites are frequently colonized, such as the hands, skin, axillae, and intestinal tract ${ }^{5}$. Nasal colonization by $S$. aureus, including MRSA, is known to be a high-risk factor for subsequent infection ${ }^{6}$.

Colonized people are usually asymptomatic, and there are three categories of MRSA carriers: noncarriers, persistent carriers (who are infected with the same strain for a long time), and intermittent carriers (who are colonized with different strains for a short time) ${ }^{7}$.

MRSA could be divided into community acquired (CA-MRSA) and hospital acquired (HA-MRSA) according to the origin of the strain. While the earlier strains mostly harm healthy young individuals through soft tissue infections, the later strains primarily affect elderly patients who are exposed to health care settings and can cause pneumonia, bacteremia, and invasive infections ${ }^{8}$.

CA-MRSA strains vary from HA-MRSA bacteria in several ways, including the formation of Panton- 
Valentine leucocidin (PVL), high-level sensitivity to non $\beta$-lactam antibiotics, and carriage of staphylococcal chromosomal cassette ( $\mathrm{SCCmec}$ ) types IV and $\mathrm{V}^{9}$.

ID-MRSA is a mecA gene-positive $S$. aureus strain that changes from a methicillin-sensitive $S$. aureus (MSSA) phenotype to a CA-MRSA phenotype after being exposed to a $\beta$-lactam drug ${ }^{10}$.

\section{METHODOLOGY}

\section{Study population:}

This study was conducted on $100 \mathrm{HCW}$ in MUCH over one year period from December 2019 till November 2020, in Medical Microbiology and immunology Department, Faculty of Medicine, Mansoura University. The protocol of this study was accepted by Institutional Review Board (IRB), Faculty of Medicine, Mansoura University; code number: MS.19.09.818.

Each participant was subjected to history taking and nasal swabs: demographic (age, gender, duration of work experience, place of work), the risk factors for MRSA colonization including antibiotic therapy in the previous 6 months before study or during study period, nasal spray use, allergic rhinitis, chronic sinusitis, asthma, eczema, diabetes mellitus, smoker, previous hospitalization, Nasal swabs were taken twice (first nasal swab from $100 \mathrm{HCWs}$ and follow up swab after three months from MRSA colonized to differentiate between transient and persistent MRSA colonization).

\section{Collection and processing of samples}

Nasal swabs were taken from $100 \mathrm{HCW}$ and were inoculated on Mannitol salt agar (MSA), Mannitol fermenting colonies that were yellow were selected and sub-cultured on Nutrient agar (NA). Colonies on NA were subjected to Gram's staining and biochemical reactions then antimicrobial susceptibility testing was performed using modified Kirby-Bauer disc diffusion method on Muller- Hinton agar using the following antibiotic discs: cefoxitin $(30 \mu \mathrm{g})$, Azithromycin $(15 \mu \mathrm{g})$, Clindamycin $(2 \mu \mathrm{g})$, Gentamicin $(10 \mu \mathrm{g})$, Trimethoprimsulfamethoxazole, Linezolid, Ciprofloxacin (5 $\mu \mathrm{g})$ and Vancomycin $(30 \mu \mathrm{g})$. Interpretation of diameter of zone of inhibition according to Clinical and Laboratory Standards Institute guidelines. Mupirocin $(200 \mu \mathrm{g})$ susceptibility test was tested on MRSA isolates interpreted according to manufacturer recommendations (resistant if $\leq 18 \mathrm{~mm}$ ).

\section{DNA extraction for PCR:}

DNA was extracted from fresh growth. One to five isolated bacterial colonies were suspended in $50 \mu \mathrm{l}$ of sterile distilled water and heated for ten minutes at $99^{\circ} \mathrm{C}$. Five $\mu \mathrm{l}$ of supernatant were used as PCR templates after centrifugation at $30,000 \times \mathrm{g}$ for 1 minute $^{11}$

Genetic characterization of MRSA isolates by detection of SCCmec IV and Panton-Valentine Leukocidin (PVL) genes by PCR.

Table 1: primers and amplicon size of the genes:

\begin{tabular}{|l|l|c|}
\hline Gene & Primer sequence (5' to 3') & Amplicon size (bp) \\
\hline SCCmec Type IV (ccrB2) (F) & CGAACGTAATAACATTGTCG & 203 \\
\hline SCCmec Type IV (ccrB2) (R) & TTGGCWATTTTACGATAGCC & \\
\hline Luk-PV-1 (F) & ATCATTAGGTAAAATGTCTGGACATGATCCA & 433 \\
\hline Luk-PV-2 (R) & GCATCAAGTGTATTGGATAGCAAAAGC & \\
\hline
\end{tabular}

Cycling conditions for SCCmec Type IV(ccrB2) gene according to (Milheiriço et al) ${ }^{12}$, cycling conditions for PVL gene according to (Ul Bashir) ${ }^{13}$.

Detection of mec A gene in MSSA isolates:

Table 2: Primers and amplicon size of the gene:

\begin{tabular}{|l|l|c|}
\hline Gene & Primer sequence (5' to 3') & Amplicon size (bp) \\
\hline mecA (F) & GTG GAA TTG GCC AATACA GG & 1339 \\
\hline$(\mathrm{R})$ & TGA GTT CTG CAG TAC CGG AT & \\
\hline
\end{tabular}

Cycling conditions according to (UI Bashir) ${ }^{\mathbf{1 3}}$.

Agarose gel electrophoresis (1.5\% agarose) was done for detection of the amplicons.

The lengths of the PCR products were estimated by comparison with the $100 \mathrm{bp}$ DNA ladder molecular size markers (Promega), ${ }^{14}$. 


\section{Statistical analysis}

Data were analyzed using the Statistical Package of Social Science (SPSS) program for Windows (Standard version 21). The normality of data was first tested with one-sample Kolmogorov-Smirnov test. Qualitative data were described using number and percent. Association between categorical variables was tested using Fischer exact test when expected cell count less than 5 . Continuous variables were presented as mean \pm SD (standard deviation) for normally distributed data and median (min-max) for non-normal data. For all above mentioned statistical tests done, the threshold of significance was fixed at $5 \%$ level.The results were considered significant when $\mathrm{p} \leq 0.05$. The smaller the $\mathrm{p}$ value obtained, the more significant are the results.

\section{RESULTS}

Overall prevalence of nasal carriage of MRSA among HCWs was $25 \%$. On testing antibiotic susceptibility pattern of the MRSA isolates by Disk Diffusion method: The highest resistance rates were for Sulphamethoxazole/Trimethoprim (76\%) and Gentamycin (68\%) followed by Clindamycin (56\%) and Azithromycin (52\%). Linezolid (44\%) and Ciprofloxacin (36\%). The least resistance was to Mupirocin (16\%) and to Vancomycin $(0 \%)$ as showed in table 3.

Table 3: Antibiotic Susceptibility pattern of MRSA isolates by Disk Diffusion method (n=25):

\begin{tabular}{|l|c|c|c|}
\hline \multicolumn{1}{|c|}{ Antibiotics resistance } & Sensitive & Intermediate resistant & Resistance \\
\hline Mupirocin & $21(84.0 \%)$ & $0(0 \%)$ & $4(16.0 \%)$ \\
\hline Gentamycin & $6(24.0 \%)$ & $2(8.0 \%)$ & $17(68.0 \%)$ \\
\hline Azithromycin & $10(40.0 \%)$ & $2(8.0 \%)$ & $13(52.0 \%)$ \\
\hline Vancomycin & $17(68.0 \%)$ & $8(32.0 \%)$ & $0(0 \%)$ \\
\hline Sulphamethoxazole/Trimethoprim & $6(24.0 \%)$ & $0(0 \%)$ & $19(76.0 \%)$ \\
\hline Linezolid & $14(56.0 \%)$ & $0(0 \%)$ & $11(44.0 \%)$ \\
\hline Clindamycin & $9(36.0 \%)$ & $2(8.0 \%)$ & $14(56.0 \%)$ \\
\hline Ciprofloxacin & $11(44.0 \%)$ & $5(20.0 \%)$ & $9(36.0 \%)$ \\
\hline
\end{tabular}

Non-Significant associations between nasal carriage of MRSA and demographic data.

As regard to risk factors among MRSA colonizers and non-colonizer groups except for diabetes mellitus, there was no significant association between all risk factors and MRSA nasal colonization as showed in table 4.

Table 4: Risk factors among MRSA colonizers and Non colonizer groups:

\begin{tabular}{|l|c|c|c|}
\hline \multicolumn{1}{|c|}{ Risk Factors } & $\begin{array}{c}\text { MRSA colonizers } \\
(\mathbf{n = 2 5})\end{array}$ & $\begin{array}{c}\text { Non colonizer } \\
(\mathbf{n}=75)\end{array}$ & $\begin{array}{c}\chi^{2} \\
(\mathbf{P} \text { value })\end{array}$ \\
\hline Intake of antibiotic therapy in the last 6 months. & $10(40.0 \%)$ & $29(38.7 \%)$ & $\begin{array}{c}\chi^{2}=0.014 \\
\mathrm{P}=0.906\end{array}$ \\
\hline Using Nasal spray. & $2(8.0 \%)$ & $3(4.0 \%)$ & $\begin{array}{c}\text { FET } \\
\mathrm{P}=0.598\end{array}$ \\
\hline Presence of allergic rhinitis. & $6(24.0 \%)$ & $12(16.0 \%)$ & $\begin{array}{c}\chi^{2}=0.813 \\
\mathrm{P}=0.367\end{array}$ \\
\hline Presence of chronic sinusitis. & $6(24.0 \%)$ & $11(14.7 \%)$ & $\begin{array}{c}\chi^{2}=1.16 \\
\mathrm{P}=0.282\end{array}$ \\
\hline Presence of asthma. & $4(16.0 \%)$ & $10(13.3 \%)$ & $\begin{array}{c}\chi^{2}=0.111 \\
\mathrm{P}=0.739\end{array}$ \\
\hline Presence of eczema. & $2(8.0 \%)$ & $8(10.7 \%)$ & $\begin{array}{c}\chi^{2}=0.148 \\
\mathrm{P}=0.70\end{array}$ \\
\hline Presence of diabetes mellitus. & $4(16.0 \%)$ & $3(4.0 \%)$ & $\begin{array}{c}\chi^{2}=4.15 \\
\mathrm{P}=0.042^{*}\end{array}$ \\
\hline Smoking. & $2(8.0 \%)$ & $4(5.3 \%)$ & $\begin{array}{c}\mathrm{FET} \\
\mathrm{P}=0.638\end{array}$ \\
\hline Prior hospitalization. & & & $\begin{array}{c}\chi^{2}=1.44 \\
\mathrm{P}=0.229\end{array}$ \\
\hline
\end{tabular}

Prevalence of CA-MRSA strains among MRSA nasal colonization group(n=25): $4(16.0 \%)$ were Sccmec IV and PVL Positive CA-MRSA strains while 21(84\%) HA-MRSA.

As regard follow up of MRSA nasal colonization group after three months: persistent MRSA carriers accounted for $52 \%$ of previously colonized HCWs, whereas transient carriers accounted for $48 \%$. 
Table 5: Association between CA - MRSA, HA - MRSA and risk factors:

\begin{tabular}{|c|c|c|c|}
\hline Risk Factors & $\begin{array}{c}\mathrm{CA} \text { - MRSA } \\
(\mathrm{n}=4)\end{array}$ & $\begin{array}{c}\text { HA- MRSA } \\
(n=21)\end{array}$ & p value \\
\hline Intake of antibiotic therapy in the last 6 months & $2(50.0 \%)$ & $8(38.1 \%)$ & 1.0 \\
\hline Using nasal spray & $2(50.0 \%)$ & $0(0 \%)$ & $0.02 *$ \\
\hline Presence of allergic rhinitis & $2(50.0 \%)$ & $4(19.0 \%)$ & 0.234 \\
\hline Presence of chronic sinusitis & $2(50.0 \%)$ & $4(19.0 \%)$ & 0.234 \\
\hline Presence of asthma & $2(50.0 \%)$ & $2(9.5 \%)$ & 0.106 \\
\hline Presence of eczema & $2(50.0 \%)$ & $0(0 \%)$ & $0.02 *$ \\
\hline Presence of diabetes mellitus & $2(50.0 \%)$ & $2(9.5 \%)$ & 0.106 \\
\hline Smoking & $2(50.0 \%)$ & $0(0 \%)$ & $0.02 *$ \\
\hline
\end{tabular}

*Significant $\mathrm{p} \leq 0.05$

Eczema, smoking and nasal spray usage were significant risk factors for CA-MRSA nasal colonization; however, they were not significant for HA-MRSA nasal colonization.

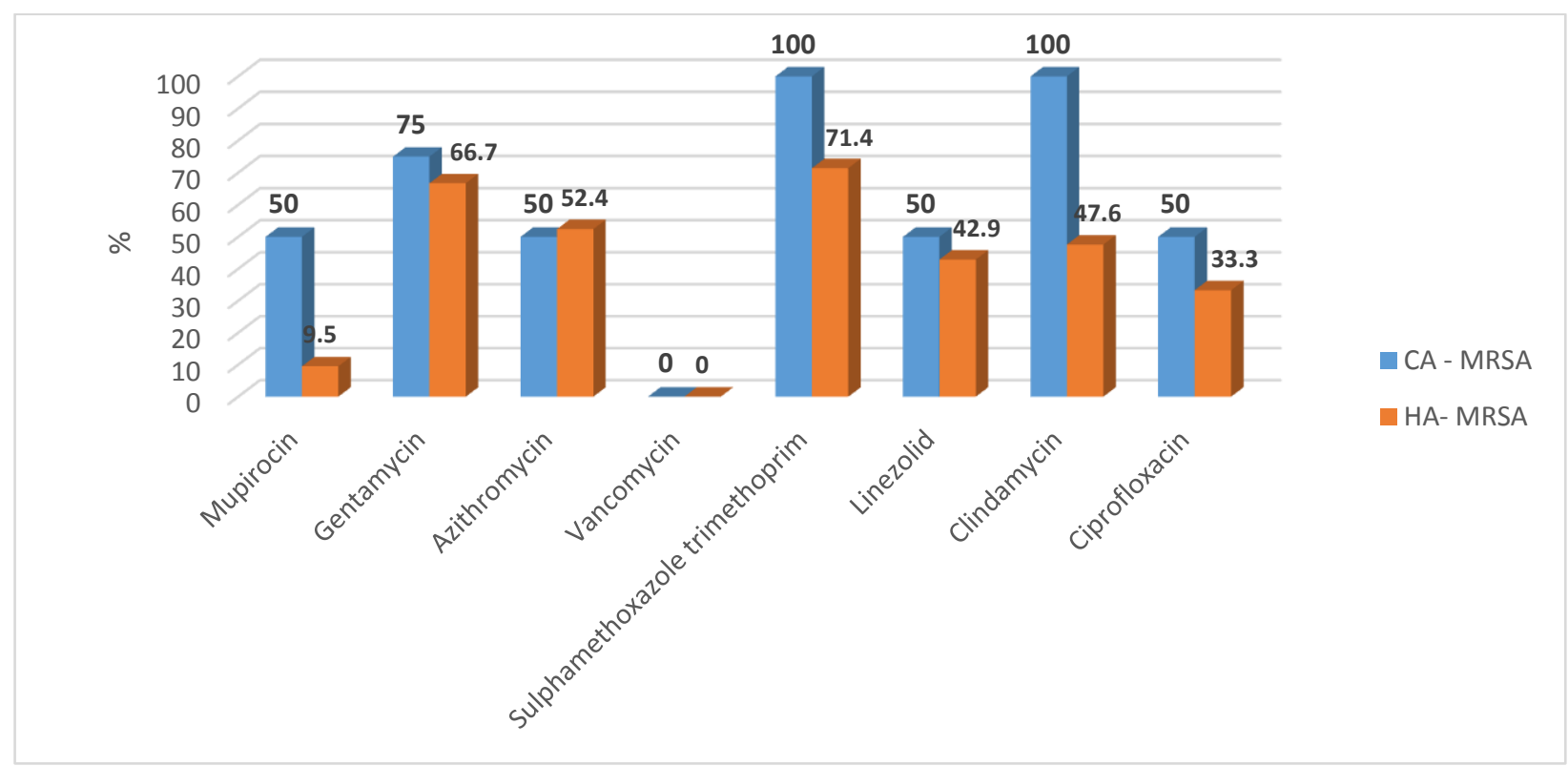

Figure 1: Antibiotic resistance in CA - MRSA and HA-MRSA

CA-MRSA was more resistant than HA-MRSA as shown in figure1.

MSSA were all mecA negative, and there was no inducible dormant MRSA.

\section{DISCUSSION}

The total prevalence of nasal carriage of MRSA among HCWs in this study was $25 \%$. This was similar to the result from El Aila et al. ${ }^{1}$ in Gaza Strip which was $(25.5 \%)$ and near to the prevalence reported by Abdel Rahman et al., ${ }^{15}$ in Egypt $(20 \%)$. Higher prevalence was detected in Nigeria $(39.9 \%)$ by Fadeyi et al., ${ }^{\mathbf{1 6}}$. While lower prevalence reported from Ethiopia (14.1\%) by Gebreyesus et al. ${ }^{17}$.

The disparities in prevalence between research regions might be related to variances in patient admission rates, study durations, microbiological procedures (from sample size to culture medium), antibiotic policy, hospital and health-care worker awareness of MRSA infection control measures.

As regard antibiotic sensitivity of MRSA isolates, Mupirocin and Vancomycin were the most effective against MRSA isolates, where their susceptibility rates were $84 \%$ and $68 \%$ respectively followed by Linezolid $(56 \%)$ and Ciprofloxacin (44\%). This suggests that these antibiotics might be used to treat MRSA infections empirically. The findings were consistent with those published by Pourramezan et al., ${ }^{18}$, who found that 
Mupirocin susceptibility rates for MRSA carriers from HCWs were $83.4 \%$.

Due to widespread usage of Mupirocin ointment for skin and soft tissue infections, in decolonization programs and due to MRSA being a prominent risk factor for Mupirocin resistance, higher resistance rates reported by Madhumati et al., ${ }^{19}$ and Antonov et al., ${ }^{20}$ $(24 \%)$ and $(55.4 \%)$ were Mupirocin resistant MRSA. however, lower resistance rate $(2 \%)$ was reported by Singh et al., ${ }^{\mathbf{2 1}}$. Also Helal et al., ${ }^{\mathbf{2 2}}$ announced that all the MRSA recovered from $\mathrm{HCWs}$ were resistant to Sulphamethoxazole/Trimethoprim, these results are to some extent near to our result.

In the current study unexpected decline in Linezolid sensitivity rate was observed which may be due to its abuse in the market in treatment of MRSA infections instead of Vancomycin which is a very alarming sign.

Except for Vancomycin, which is the treatment of choice in MRSA, there is a lot of variation in antibiotic susceptibility patterns of MRSA isolates owing to diverse populations and localities, as shown in the previous data.

As regard risk factors of MRSA nasal colonization in the present study except for diabetes mellitus, there was no significant association between all risk factors and MRSA nasal colonization. Similarly in a study by Legese et al., ${ }^{23}$ percentage of MRSA colonization of the anterior nares was high among diabetic health care personnel. This might be attributed to diabetes patients' weakened immunity and in another study by Verwer et al., ${ }^{24}$ he observed that $5.8 \%$ of MRSA colonized HCWs had taken antibiotics in the preceding period.

In contrast of our findings, $\mathrm{Wu}$ et al., ${ }^{25}$ found that there were no statistically significant relationships between risk variables and nasal MRSA colonization in HCWs. Pourramezan et al., ${ }^{\mathbf{1 8}}$ could not detect any significant connections between nasal carriage of MRSA and recent antibiotic usage, but other risk variables such as dermatitis $(25 \%)$, sinusitis or rhinitis $(29.6 \%)$, diabetes mellitus (20\%), and smoking $(27.2 \%)$ were shown to be significant which partially in agreement with us.

As regard molecular characterization in our research four of the twenty-five MRSA strains tested positive for SCCmec IV as well as the PVL gene, indicating that 16 $\%$ are CA-MRSA strains and $84 \%$ are HA-MRSA strains. Higher prevalence reported by BuenaventuraAlcazaren et al. ${ }^{6}$ who observed that the prevalence of positive PVL-SCCmec IV community acquired MRSA isolates from $\mathrm{HCWs}$ was $33 \%$. However, lower prevalence documented by Preeja et al., ${ }^{\mathbf{2 6}}$ who found that $12.3 \%$ of MRSA isolates were PVL-SCCmec IV community acquired MRSA.

The findings of the molecular characterization revealed that the frequency of CA-MRSA in hospitals is growing. Patients, MRSA carrier visitors, and all medical staff might all be sources of CA-MRSA in the hospital setting.

As regard follow up of MRSA nasal colonization group after three months in the current study, persistent MRSA carriers accounted for $52 \%$ of previously colonized HCWs, whereas transient carriers accounted for $48 \%$. Higher percentage $(67.6 \%)$ of persistent MRSA colonized HCWs After three months reported by Verwer et al., ${ }^{24}$ and (32.4\%) were transiently colonized. Another higher percentage $(54 \%)$ founded by Cookson et al. ${ }^{27}$ while transient nasal carriage was (46\%). Although persistent MRSA carriage predominate in several investigations, other research founded larger proportions of transitory or intermittent carriage and other founded equivalent proportions of persistent and non-persistent carriage ${ }^{28}$.

The frequency and timing of follow-up cultures necessary are still debatable. They are determined by the aims of decolonization therapy, as well as the prevalence of MRSA in the area and the risk of reinfection. ${ }^{7}$.

Our research found that CA-MRSA were more resistant than HA-MRSA. CA-MRSA isolates were found resistant to three or more classes of antibiotics (Sulphamethoxazole/Trimethoprim, Clindamycin and Gentamycin) (MDR CA-MRSA).

Like our findings, CA-MRSA isolates containing the SCCmec type IV demonstrated stronger antibiotic resistance than HA-MRSA according to Preeja et al., ${ }^{26}$ and CA-MRSA isolates were shown to be resistant to three or more antibiotic classes. MDR CA-MRSA has been reported worldwide as mentioned by Earls et al., ${ }^{29}$ and Lee et al. ${ }^{30}$.

In contrast to our findings Vysakh and Jeya, ${ }^{\mathbf{3 1}}$ and Fey et al., ${ }^{32}$ found that CA-MRSA is more sensitive to non $\beta$-lactam antibiotics than HA-MRSA.

From all these studies we suppose that CA-MRSA isolated from HCWs with MDR pattern can lead to the spread of multidrug-resistant virulent strains of CAMRSA in the hospital and the community.

This study founded that Eczema, nasal spray usage, and smoking are all major risk factors for CA-MRSA nasal colonization, however there is no link between HA-MRSA nasal colonization and any of these characteristics. Ong, ${ }^{33}$ reported that the percentage of CA MRSA colonization in patients of atopic dermatitis was from $11 \%$ to $34 \%$ also in recent years, MRSAinduced eczema aggravation has become a substantial clinical concern.

This study reported that MSSA were all mecA negative, and there was no ID MRSA. However, Kampf et al., ${ }^{10}$ reported the rate of dormant MRSA was (1.6\%)in HCWs, The rate of dormant MRSA was higher in ICU $(1.9 \%)$ compared with the general wards $(0.7 \%)$. Because the pathogen is not identified as oxacillinresistant, but may develop phenotypic resistance during antibiotic therapy, transfer of dormant MRSA to a 
patient may be even more harmful for the patient than transmission of MRSA ${ }^{15}$.

\section{CONCLUSION}

MDR CA-MRSA nasal colonization occurs in a considerable proportion of HCWs in MUCH. High priority should be given for regular screening of them and also decolonization strategy should be applied.

This manuscript has not been previously published and is not under consideration in the same or substantially similar form in any other reviewed media. I have contributed sufficiently to the project to be included as author. To the best of my knowledge, no conflict of interest, financial or others exist. All authors have participated in the concept and design, analysis, and interpretation of data, drafting and revising of the manuscript, and that they have approved the manuscript as submitted.

\section{REFERENCES}

1. El Aila NA, Al Laham NA, Ayesh BM. Nasal carriage of methicillin resistant Staphylococcus aureus among health care workers at Al Shifa hospital in Gaza Strip. BMC Infect Dis. 2017;17(28):1-7.

2. Krismer B, Weidenmaier C, Zipperer A, Peschel A. The commensal lifestyle of Staphylococcus aureus and its interactions with the nasal microbiota. Nat Rev Microbiol. 2017; 15(11):675-87.

3. Garcia C, Acunặ-Villaordunặ A, Dulanto A, Vandendriessche $\mathrm{S}$, Hallin $\dot{\mathrm{M}}$, Jacobs $\mathrm{J}$, et al. Dynamics of nasal carriage of methicillin-resistant Staphylococcus aureus among healthcare workers in a tertiary-care hospital in Peru. Eur $\mathrm{J}$ Clin Microbiol Infect Dis. 2016; 35(1):89-93.

4. Singh S, Malhotra R, Grover P, Bansal R, Galhotra S, Kaur R, et al. Antimicrobial resistance profile of Methicillin-resistant Staphylococcue aureus colonizing the anterior nares of health-care workers and outpatients attending the remotely located tertiary care hospital of North India. J Lab Phys. $2017 ; 9(4): 317-21.7$.

5. Acton DS, Plat-Sinnige MJ, van Wamel W, de Groot N, van Belkum A. Intestinal carriage of Staphylococcus aureus: how does its frequency compare with that of nasal carriage and what is its clinical impact? Eur J Clin Microbiol Infect Dis. 2009; 28:115-127.

6. Buenaventura-Alcazaren FA, dela Tonga A, OngLim A, Destura RV. Prevalence and molecular characteristics of MRSA nasal carriage among hospital care workers in a tertiary hospital in the
Philippines. Journal of Microbiology, Immunology and Infection. 2020; 53(5), pp.739-745.

7. Albrich WC, Harbarth S. Health-care workers: source, vector, or victim of MRSA? Lancet Infect Dis.2008; 8:289-301.

8. David MZ, Daum RS. 'Community-associated methicillin-resistant Staphylococcus aureus: epidemiology and clinical consequences of an emerging epidemic', Clinical microbiology reviews.2010; 23(3), pp. 616-687.

9. Figueiredo AM. What is behind the epidemiological difference between communityacquired and health-care associated methicillinresistant Staphylococcus aureus? Virulence. 2017; 8(6), pp.640-642.

10. Kampf G, Adena S, Ru“ den H, Weist K. Inducibility and potential role of mecAgenepositive oxacillin-susceptible Staphylococcus aureus from colonized healthcare workers as a source for nosocomial infections. $\mathrm{J}$ Hosp Infect.2003;54:124-9.

11. Zhang K, McClure JA, Elsayed S, Louie T, Conly JM. Novel multiplex PCR assay for characterization and concomitant subtyping of staphylococcal cassette chromosome mec types I to $\mathrm{V}$ in methicillin-resistant Staphylococcus aureus. Journal of clinical microbiology. 2005; 43(10), pp.5026-5033..

12. Milheiriço C, Oliveira DC, de Lencastre $H$. 'Multiplex PCR strategy for subtyping the staphylococcal cassette chromosome mec type IV in methicillin-resistant Staphylococcus aureus:"SCC mec IV multiplex",, Journal of antimicrobial chemotherapy. 2007;60(1), pp. 4248.

13. Ul Bashir Y. 'SCCmec Type IV and V Methicillin Resistant Staphylococcus aureus Intrusion in Healthcare Settings. American Journal of Clinical Microbiology and Antimicrobials. 2019; pp. 1-5.

14. Arciola CR, Baldassarri L, Montanaro L. 'Presence of icaA and icaD genes and slime production in a collection of staphylococcal strains from catheterassociated infections', Journal of clinical microbiology. 2001; 39(6), pp. 2151-2156.

15. Abdel Rahman AT, Hafez SF, Abdelhakam SM, Ali-Eldin ZA, Esmat IM, Elsayed MS, et al. 'Antimicrobial resistant bacteria among health care workers in intensive care units at Ain Shams University Hospitals.', Journal of the Egyptian Society of Parasitology.2010; 40(1), pp. 71-83.

16. Fadeyi A, Bolaji BO, Oyedepo OO, Adesiyun OO, Adeboye MAN, Olanrewaju TO, et al. 'Methicilin resistant Staphylococcus aureus carriage amongst healthcare workers of the critical care units in a 
Nigerian hospital', Am J Infect Dis.2010; 6(1), pp. 18-23.

17. Gebreyesus A, Gebreselassie S, Mihret A. 'Nasal and hand carriage rate of methicillin resistant Staphylococcus aureus (MRSA) among health care workers in Mekelle hospital, North Ethiopia'. Ethiop Med J. 2013;51(1):41-7.

18. Pourramezan N, Moghadam SO, Pourmand MR. Methicillin-resistant Staphylococcus aureus tracking spread among health-care workers and hospitalized patients in critical wards at a university hospital, Tehran, Iran. New microbes and new infections.2019; 27, pp.29-35.

19. Madhumati B, Rajendran V, Ashwin K. Prevalence of mupirocin resistance in methicillin resistant Staphylococcus aureus strains isolated from a tertiary care hospital. Int J Curr Microbiol App Sci.2018; 7(5), pp.329-36.

20. Antonov NK, Garzon MC, Morel KD, Whittier S, Planet PJ, Lauren CT. High prevalence of mupirocin resistance in Staphylococcus aureus isolates from a pediatric population. Antimicrobial agents and chemotherapy. 2015;59(6), pp.33503356.

21. Singh AK, Venkatesh V, Singh M.'Mupirocin resistance in clinical isolates of Staphylococcus aureus in a tertiary care hospital set up in North India', International Journal of Medical Research \& Health Sciences.2013;2(4), pp. 840-847.

22. Helal ZH, Mohamed HE, ElMahallawy HA, Afifi SS. Regular article molecular characterization and antimicrobial susceptibility of mrsa isolated from chronic hemodialysis outpatients and their correlation to mrsa colonization among healthcare workers. Bacterial Empire.2019; 2( 4), 70-75

23. Legese H, Kahsay AG, Kahsay A, Araya $T$, Adhanom G, Muthupandian S, Gebreyesus A. Nasal carriage, risk factors and antimicrobial susceptibility pattern of methicillin resistant Staphylococcus aureus among healthcare workers in Adigrat and Wukro hospitals, Tigray, Northern Ethiopia. BMC research notes. 2018; 11(1), pp.1-6..

24. Verwer PEB, Robinson JO, Coombs GW, Wijesuriya T, Murray RJ, Verbrugh HA, Riley T, Nouwen JL. Christiansen KJ. Prevalence of nasal methicillin-resistant Staphylococcus aureus colonization in healthcare workers in a Western Australian acute care hospital. European journal of clinical microbiology \& infectious diseases. 2012; 31(6), pp.1067-1072.

25. Wu TH, Lee CY, Yang HJ, Fang YP, Chang YF, Tzeng SL, Lu MC. Prevalence and molecular characteristics of methicillin-resistant Staphylococcus aureus among nasal carriage strains isolated from emergency department patients and healthcare workers in central Taiwan. Journal of Microbiology, Immunology and Infection. 2019; 52(2), pp.248-254.

26. Preeja PP, Kumar SH, Shetty V. 'Prevalence and characterization of methicillin-resistant staphylococcus aureus from community-and hospital-associated infections: A tertiary care center study', Antibiotics. 2021; 10(2), pp. 1-10. doi: 10.3390/antibiotics 10020197.

27. Cookson BBMIMW, Peters B., Webster M, Phillips I, Rahman M, Noble W. Staff carriage of epidemic methicillin-resistant Staphylococcus aureus. Journal of clinical microbiology. 1989; 27(7), pp.14711476.

28. Regev-Yochay G, Rubinstein E, Barzilai A, Carmeli Y, Kuint J, Etienne J, Blech M, Smollen G, Maayan-Metzger A, Leavitt A, Rahav G. Methicillin-resistant Staphylococcus aureus in neonatal intensive care unit. Emerging infectious diseases. 2005;11(3), p.453.

29. Earls MR, Kinnevey PM, Brennan GI, Lazaris A, Skally M, O'Connell B, Humphreys H, Shore AC, Coleman DC. The recent emergence in hospitals of multidrug-resistant community-associated sequence type 1 and spa type t127 methicillin-resistant Staphylococcus aureus investigated by wholegenome sequencing :Implications for screening. PLoS One.2017; 12(4),p.e0175542.

30. Lee GC, Dallas SD, Wang Y, Olsen RJ, Lawson KA, Wilson J, Frei CR. Emerging multidrug resistance in community-associated Staphylococcus aureus involved in skin and soft tissue infections and nasal colonization. Journal of Antimicrobial Chemotherapy. 2017;72(9), pp.2461-2468.

31. Vysakh PR, Jeya M. 'A comparative analysis of community acquired and hospital acquired methicillin resistant Staphylococcus aureus, Journal of clinical and diagnostic research: JCDR. 2013; 7(7), p. 1339.

32. Fey PD, Said-Salim B, Rupp ME, Hinrichs SH, Boxrud DJ, Davis CC, Kreiswirth BN, Schlievert PM. Comparative molecular analysis of community-or hospital-acquired methicillinresistant Staphylococcus aureus. Antimicrobial agents and chemotherapy. 2003;47(1), pp.196-203..

33. Ong PY. 'Recurrent MRSA skin infections in atopic dermatitis', The Journal of Allergy and Clinical Immunology: In Practice. 2014; 2(4), pp. 396-399. 